\title{
A young patient with perinatal HIV infection treated for 17 consecutive years with antiretroviral therapy: extremely severe lipo-accumulation picture
}

\author{
Roberto Manfredi \\ From $16^{\text {th }}$ International Symposium on HIV and Emerging Infectious Diseases \\ Marseille, France. 24-26 March 2010
}

\section{Background}

Like adults, also pediatric HIV-infected patients (p) are prone to suffer from a lipodystrophy syndrome, usually accompanied by dysmetabolic disorders. The psychological consequences of the morphologic changes are expected to be particularly devastating in development ages.

\section{Methods}

Like adults, also pediatric HIV-infected patients (p) are prone to suffer from a lipodystrophy syndrome, usually accompanied by dysmetabolic disorders. The psychological consequences of the morphologic changes are expected to be particularly devastating in development ages.

\section{Results}

Notwithstanding a cumbersome anti-HIV management, the immune-virological situation remained under control (the last laboratory assays showed a viremia of 1,300 HIV-RNA copies/mL, and a CD4+ count of 612 cells $/ \mu \mathrm{L}$ ), and our p never suffered from AIDS-related complications. Unfortunately, starting 7 years ago, a bilateral symmetric lipomastia appeared, associated in the past two years with a bilateral, dorsal lipid accumulation (both confirmed by ultrasonography), which were responsible for severe psychological disturbances. Hormonal-metabolic workup failed in disclosing relevant abnormalities, as to serum FSH, LH, testosteron, estradiol, estrone, progesteron, dehydroepiandrosterone, and

Correspondence: Roberto.manfredi@unibo.it

Infectious Diseases, University of Bologna, S. Orsola Hospital, Bologna, Italy prolactin levels, thyroideal profile (TSH, fT3, fT4, and anti-thyroid antibodies), as well as metabolic pattern (fasting glucose, total cholesterolemia, HDL- and LDLfractions, apolipoproteins, C-peptide, insulin, fruttosamin, glycosilated hemoglobin, lactate, and bicarbonate), when excluding a moderate hypertriglyceridemia.

\section{Discussion}

While in adult $\mathrm{p}$ the prolonged course of HIV infection and combination antiretroviral therapy (cART) usually leads to an associated dysmetabolic sindrome and lipodystrophy, characterized by co-existing peripheral lipoatrophy and visceral adiposity, usually accompanied by a mixed dyslipidemia and insulin resistance, our young $\mathrm{p}$ surprisingly developed an isolated mammary and dorsal lipid hyperaccumulation syndrome with lipomastia prevailing over gynecomastia. Also the recent advice to further modify cART (by including NNRTIs), is not expected to act significantly in short-mid term on the particular, focal lipoaccumulation features of our young p. A surgical option (liposuction), is the most reliable clinical option.

Published: 11 May 2010

\section{doi:10.1186/1742-4690-7-S1-P67}

Cite this article as: Manfredi: A young patient with perinatal HIV infection treated for 17 consecutive years with antiretroviral therapy: extremely severe lipo-accumulation picture. Retrovirology 2010 7(Suppl 1): P67. 